\title{
Questionnaire on Mothers' Cultural Beliefs About Weaning: Development and Psychometric Evaluation
}

\author{
Zahra Jannat-Alipoor $^{\mathbb{1}}$, Nasrin Navabi ${ }^{1}$, Abbas Ebadi $^{2}$, Fatemeh Ghaffari ${ }^{{ }^{*}}$
}

\begin{abstract}
Objectives: This study was designed to investigate the role of cultural beliefs on weaning. Therefore, the obtained results can be used for health policymakers who design strategies to prevent physical and mental damage to mothers and their children. The main objective of this study was to design a questionnaire probing the role of cultural beliefs on weaning.

Materials and Methods: The sequential combination exploratory mixed methods design was used to develop the questionnaire format in two sections. The qualitative section was designed to probe the role of cultural beliefs on weaning, which included a literature and related tools review and fieldwork (semi-structured interviews with mothers). Twenty-two studies were examined in the literature review and 14 mothers were selected and interviewed by a purposive sampling technique. The interviews continued up to data saturation as well. In addition, data analyses for both steps were conducted using conventional and textual content analyses. The quantitative section was a methodology study that was accomplished in two parts. Questionnaire items were formed using the data and item pool obtained from the first part. Finally, the psychometric properties of the questionnaire were checked using face, content, and construct validity, followed by probing reliability using Cronbach's alpha reliability in the second part.

Results: Qualitative data analysis results were organized according to the foundational issues regarding the need for weaning, attitude toward weaning, awareness about weaning, society's culture, health literacy, self-action, others' experiences, professional help, family members' support, and the outcomes. The item pool was formed using literature reviews and interviews. A 49-item questionnaire was developed after the completion of the psychometric process. The Kaiser-Meyer-Olkin Index of Sampling Adequacy and Bartlett's test of sphericity showed good results in this regard. Five components from the exploratory content analysis encompassing contexts, solutions, searching for help, maternal outcomes, and child-related outcomes demonstrated a $62.112 \%$ variance. Further, the Cronbach's alpha was 0.88 and the interclass correlation coefficient was 0.89 based on responses to the items over two administrations of the questionnaire $(P<0.001)$. Thus, these results showed a high level of tool stability.

Conclusions: For this study, a questionnaire was developed for understanding the role of cultural beliefs on weaning. It can be used for educational, research, and treatment purposes as a tool with appropriate validity and reliability, as well as short, easy, and grammatically simple items. Eventually, the questionnaire is useful for examining mothers' false beliefs about weaning and their educational needs since false beliefs could cause destructive and health-threatening behaviors.

Keywords: Cultural beliefs, Weaning, Reliability, Validity, Psychometric testing
\end{abstract}

\section{Introduction}

Breast milk is known as the best and most complete source of nutrition for newborns and children in different cultures. In the relevant literature, different ages are suggested for weaning (1). However, the need for a return to work, (2), as well as illness and disease of the mother or child, cultural contexts, misconceptions, and the level of parents' awareness may affect the age for a child for weaning (3-5). Most mothers generally receive the necessary education and consultation about breastfeeding but not weaning (6).

Mothers' conceptions about weaning are influenced by their experiences and cultural beliefs, which also influence the method of weaning. Therefore, weaning is performed in different ways and is affected by numerous factors including the education level, along with economic, social, and cultural factors (7). Cultural factors, especially, play an important role in this respect (8). Some studies showed that mothers employ different methods for weaning such as changing the breasts' appearance or using chemicals and medicines for changing the taste of breast milk $(8,9)$. Using such methods, which have cultural roots, may have physical and psychological consequences for the child such as trauma, regressive behaviors, the deprivation of maternal emotions and love, anxiety, restlessness, and sleeping disorders, as well as the feelings of less intimacy, (11) the loss of feelings, sadness, and guilt in mothers $(12,13)$. Despite these consequences, few studies point to the role of cultural beliefs in the selection of a weaning method (10). Additionally, some studies examined the qualitatively cultural beliefs of parents, especially mothers about weaning $(14,15)$. 
Although some tools were designed to study this subject, they were not tested psychometrically to the extent needed for gathering all related aspects. Therefore, designing a tool for finding a mother's approach, which is tested psychometrically, toward weaning seems essential. Using mixed methods for profiting from both qualitative and quantitative data can result in obtaining a precise image of the phenomenon as well. Designing a tool using such methods can also help midwives, nurses, and other health providers in applying accurate educational intervention for directing mothers toward healthy behaviors (16). Health policymakers can further benefit from this study as they design new strategies for preventing mental and physical damage caused by weaning.

\section{Materials and Methods}

The inductive-sequential mixed-method design was adopted to develop the questionnaire used in this study, including a combination of qualitative and quantitative measures obtained through ethnographic methods in the fieldwork $(17,18)$.

\section{Step One: Qualitative Study}

The mothers' conceptions of the weaning phenomenon were probed in two parts including an examination of the literature and related tools and fieldwork (semi-structured interviews with mothers) as the qualitative step. Vast searches were conducted for clarifying the concept of weaning and the role of cultural beliefs on the subject in the literature review. Several keywords such as "cultural beliefs", "weaning", tool design", and "psychometric tests" were searched in SCOPUS, Ovid, Proquest, Science Direct, PubMed, and CINHAL databases without setting any specific time limit for the literature search. Finally, 22 papers were selected for the study and a textual content analysis was used for analyzing these papers. The data analysis involved a careful examination of the papers, followed by preparing the list of items for the final data check.

Furthermore, fieldwork involved inviting mothers to health centers in the Northern provinces of Iran and a purposive sampling method was utilized to select the participants based on factors such as exclusive breastfeeding, child's gender, weaning during data gathering, willingness for participation, and sufficient communicative skills. The maximum variability of age, the number of children, educational level, occupation, and economic situation were considered as well. Moreover, detailed semi-structured interviews were used for data collection. The length for each interview relied on each participant's patience and willingness to share the experiences of understanding, methods, and outcomes of weaning and the interviews were recorded.

The interviewees were asked guided questions such as "Would you tell us how you weaned your child?", "What kind of method did you use?", "What kind of knowledge resources did you use in this regard?", "What kind of consequences did this method have for your child?", and "What factors affected you regarding using this method of weaning?". Additionally, probing questions such as "What do you mean?" or "Could you give more details" were designed if needed. Finally, participants were asked to mention any missed detail or point. Subsequently, they were informed about the possibility of further interviews.

Similarly, 30-40-minute face-to-face interviews were conducted in clinical settings. Data saturation appeared when the codes became repetitive and no new category or subcategory was created after conducting 14 interviews with mothers who met the inclusion criteria. A qualitative content analysis was used for analyzing the data by conventionally applying the Granheim and Lundman's pattern (19).

The following steps were taken to analyze the data:

- Analyzing and reading each recorded interview several times after transcribing in order to obtain a comprehensive view of the entire subject;

- Dividing the text into meaning units and labelling them with the codes;

- $\quad$ Sorting the codes into subcategories and categories based on comparisons regarding their similarities and differences;

- Formulating the themes as the expression of the latent content of the text.

\section{Trustworthiness}

The gathered data in the qualitative step were checked carefully to fit the Guba and Lincoln criteria for credibility, dependability, confirmability, and transferability (20). Further, peer and member checks were used in this step. To validate the combination study, investigators who dealt with threats regarding data collection in both qualitative and quantitative studies included different participants. Sufficient sample size was applied to obtain data saturation in the qualitative step although a larger size was used in the quantitative step for better validation.

\section{Step Two: Quantitative Study}

Inductive (items extracted from participants' experiences) and deductive (codes extracted from the literature review) methods were used in designing the tool. All related and in-access tools were checked in the literature review to avoid missing any possible items. Furthermore, researchers prepared a draft of items according to the categories and subcategories of "cultural beliefs about weaning" (Table 1).

All possible items were checked and an item pool was formed containing 64 items. Then, each item was reviewed by the research team according to the research subject in order to calculate the constructs and formal, content, and construct validity were ensured at this stage.

\section{Face Validity}

Face validity was checked via both qualitative and 
Table 1. Sample Format for Defining Questionnaire Items

\begin{tabular}{l}
\hline Comments and Experiences of Participants \\
\hline "I poured black pepper on my breast the last time my child requested breast milk. I put my breast into his mouth \\
$\begin{array}{l}\text { and it was like a bomb! He started crying and after a week, guess what? He didn't tend to want breast milk } \\
\text { anymore. That was a good method." }\end{array}$ \\
$\begin{array}{l}\text { Pouring pepper on the breast is effective for weaning. } \\
\text { Traditional }\end{array}$ \\
\hline
\end{tabular}

quantitative methods. It is considered the degree to which the questionnaire appears effective in terms of its stated aims qualitatively. Ten participating mothers were asked to comment on difficulty, relevancy, and ambiguous levels in face-to-face interviews to check the face validity qualitatively.

Moreover, the item impact method was applied to decrease or eliminate inappropriate items and determine the importance of each item as a quantitative method. The 5-point Likert-type scale ("absolutely important" to "absolutely unimportant") was used to study each item, and mothers were asked to determine the importance of each item according to their experiences. The least point value for item impact was set at 1.5 and any item receiving that score or less was eliminated from the study (21).

\section{Content Validity}

Both qualitative and quantitative methods were used to check content validity. Eight nursing lecturers with sufficient working experience in clinical, theoretical, and research settings were asked to comment on grammar, wording, allocation, and scaling of the items. Both the content validity index (CVI) and content validity ratio (CVR) were utilized to check content validity quantitatively.

\section{Content Validity Ratio}

The Lawshe model was used to determine the CVR (22) in this study. Ten subject matter experts (nursing faculty members with working experience in clinical settings, tool making, and teaching newborn and infant nursing subjects) were asked to check the necessity of the items according to a three-point scale (i.e., "necessary", "helpful but not necessary", and "unnecessary").

The CVR varied between 1 and -1 . The higher score indicated further agreement among the members of the panel on the necessity of including an item in an instrument. The applied formula was $\mathrm{CVR}=$ (ne $\mathrm{N} / 2) /(\mathrm{N} / 2)$, in which $\mathrm{Ne}$ was the number of panelists representing "essential" and $\mathrm{N}$ was the total number of panelists. The numeric value for CVR was determined using Lawshe's table.

The minimum CVR value was set at 0.62 according to Lawshe's table and the number of experts (all responded to the questions). An item was considered necessary with a statistically significant level $(P<0.05)$ if the outcome number was larger than the number of the above-mentioned table (22). The CVR was strictly used in this study, meaning that only necessary options were incorporated into the CVR formula.

\section{Content Validity Index}

Waltz and Bausell first promoted the CVI (23). Ten experts (the same people who cooperated in CVR determination) were asked to check each item for a fit with the four-point Likert-type scale (from "absolutely relevant" to "irrelevant") to determine the content validity of each item and ensure whether the items were designed properly to create the constructs.

In this study, the scale-level index (S-CVI) was calculated to find which item needed modification or elimination, whether any further items were needed to completely cover the related aspects, and to determine if all aspects of a construct were presented with a correct number of items. Accordingly, it was decided to reform or eliminate the item based on its CVI level and items with a score of 0.7 or less were eliminated from the questionnaire.

\section{Construct Validity}

Construct validity was considered in determining the efficiency of the questionnaire in the created constructs. Additionally, factor analysis was used to study the internal relationship between the variables and explore the categories of the items that were tightly related (24). Then, the Kaiser-Meyer-Olkin (KMO) and Bartlett sphericity tests were utilized before extracting the components to ensure that the items were appropriately designed for analyzing different factors.

The KMO value of 0.7 or higher was considered as the condition for sample size sufficiency in the content analysis. The Bartlett test of sphericity was used to check the level of correlation between the items of the questionnaire such that the merging of items was possible. The required sample size for content analysis varied from 5 to 10 for each item (25). In general, 310 samples were included in this study for the exploratory content analysis of the main components of the scree plot to estimate the appropriate number of factors, eigenvalues equal to and/ or more than 1, and varimax rotation. In most studies, appropriate factor loading was set as $0.4(24)$.

\section{Reliability}

The reliability of this study was checked using internal consistency and stability methods. In addition, Cronbach's alpha was used in the internal consistency method and the minimum alpha was considered to be 0.7 (26). The test- 
retest and intraclass correlation coefficient (ICC) were applied to check stability and 30 mothers were asked to complete the questionnaire twice in a two-week interval. The ICC is a reliability measure and a form of Cronbach alpha, which describes how strongly the units in the same group resemble each other. The acceptable minimum for ICC was set at 0.7 (27).

\section{Results}

A total of 409 codes were extracted in the analyzing phase, forming five categories including contexts, solutions, searching for help, as well as maternal and child-related outcomes. Additionally, 10 subcategories included the need for weaning, attitude toward weaning, awareness about weaning, society's culture, health literacy, selfaction, others' experiences, professional help, family members' support, and the outcomes.

The early item pool was designed with 64 items following the literature review. Then, ten items were analyzed qualitatively (formal validity) and no item was eliminated. However, 16 items received a score of 1.5 or less in the formal validity in/of quantitative analysis and were eliminated. Finally, a 54-item questionnaire was developed.

Ten items were reviewed in the content validity check and all possible changes were applied accordingly. The quantitative survey of content validity was based on the CVR and CVI. Three items including "scaring the child is a ruthless method for weaning", "mothers should wean in accordance with the current cultural and traditional beliefs", and "breastfeeding mothers older than 40 years old should wean earlier" were eliminated using the CVR. Two other items were also ignored after receiving a score of 0.72 and 0.73 , respectively, using the CVI. The S-CVI for all remaining items was considered as 0.93 in the next step.

A questionnaire containing 49 items based on the 5-point Likert-type scale (5 for "strongly agree" and 1 for "strongly disagree") was prepared for the exploratory content analysis phase. It was disseminated to 310 mothers who referred by health centers in Iran.

The KMO was obtained at 0.858 , which indicated sampling adequacy for per factor analysis. Furthermore, Bartlett's test of sphericity set at 7290.841 was significant at the level of 0.0001 and 525 degrees of freedom $(P=0.001)$, which justified the factor analysis based on a correlation matrix of the sample. In other words, it showed that the correlation matrix was not zero in the population.

Similarly, an analysis of the main components was used for extracting the factors in this research. A special value was used to define the number of factors. The results showed that $62.112 \%$ of the total variance was defined by four major factors. In other words, among 49 indices, there were five factors pointing to the $62.112 \%$ change in the special values of each of the text indices. Figure 1 displays the scree plot test results that determined the

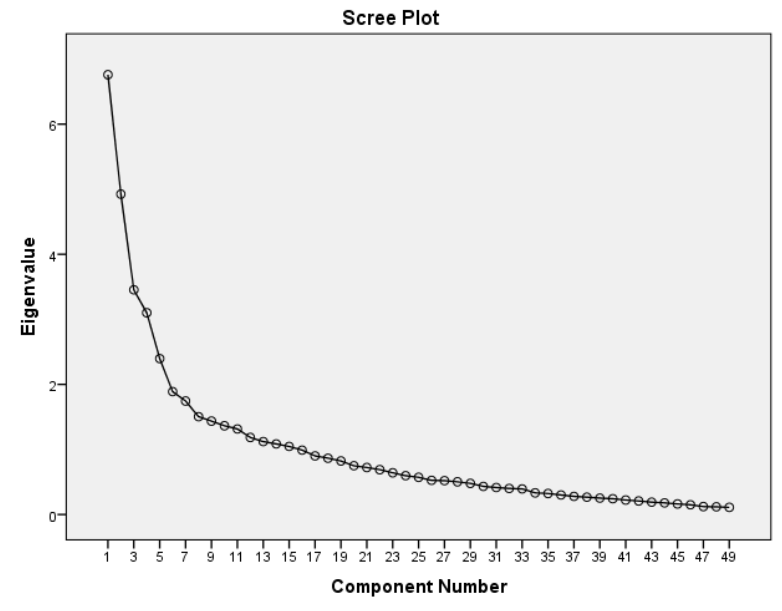

Figure 1. Scree Plot in Questionnaire Probing the Role of Cultural Beliefs on Weaning.

number of factors at this phase.

The First Component

This component included 19 items related to the contexts and the highest and the lowest load factors were observed for the following items:

"The child should be weaned if he/she is not inclined to supportive nutrition." and "The mother's occupation is effective for weaning." The variance ratio for this component was calculated as 28.904 .

The Second Component

Twelve items were included in this component stating the solutions. The highest load factor was observed for the item "Inducing the milk, which makes the child's teeth break or his/her mouth to get smelly, is effective in weaning." However, the minimum load factor was found for the item "Talking to and convincing the child is one of the weaning methods." The variance ratio for this component was computed as 17.831 .

The Third Component

This component included seven items related to searching for help by mothers. The highest load factor was related to the item "Studying related books can help mothers find the right weaning method" whereas the minimum load factor belonged to the item "Weaning is a personal issue and no consultation is needed." The variance ratio for this component was measured as 10.481 .

\section{The Forth Component}

This component encompassed 6 items related to maternal outcomes and the highest observed load factor was related to the item "Weaning results in a deep emotional distance between the mother and her child". On the other hand, the minimum load factor belonged to the item "Weaning results in a maternal feeling of pride." The variance ratio for this component was calculated as 2.949 . 


\section{The Fifth Component}

This component contained five items pointing to childrelated outcomes. The highest load factor was reported for the item "Weaning may result in unwanted habits like toe sucking, nail biting, and aggression for the child." Contrarily, the minimum load factor was related to the item "Weaning results in a better weight gain and appetite for the child."
The variance ratio for this component was estimated at 1.947.

Each component was named according to its related items after extraction. In other words, compatibility with the concepts and aspects of the mother's cultural beliefs about weaning was probed, the details of which are provided in Table 2 .

Table 2. Matrix Rotating Elements by the Varimax Method

\begin{tabular}{|c|c|c|c|c|c|}
\hline \multirow{2}{*}{ Items } & \multicolumn{5}{|c|}{ Components } \\
\hline & 1 & 2 & 3 & 4 & 5 \\
\hline Weaning gets harder as the child receives breast milk longer. & 0.476 & & & & \\
\hline Motherhood means to feed the child with breast milk as long as he/she requests it. & 0.621 & & & & \\
\hline It is possible to restart breastfeeding if weaning fails. & 0.618 & & & & \\
\hline The child reacts severely if weaning happens suddenly. & 0.498 & & & & \\
\hline The appropriate age for weaning is when the child is two years old. & 0.511 & & & & \\
\hline Weaning depends on the mother's readiness. & 0.538 & & & & \\
\hline Weaning depends on the child's readiness. & 0.508 & & & & \\
\hline The time to wean depends on the child's gender. & 0.460 & & & & \\
\hline Weaning is not affected by seasons. & 0.415 & & & & \\
\hline The child should be fed with breast milk as long as he/she tends to enjoy it. & 0.626 & & & & \\
\hline The child should be weaned when he/she reaches a certain age. & 0.519 & & & & \\
\hline The child should be weaned at any age if the mother has a low milk supply. & 0.735 & & & & \\
\hline The mother's occupation has an effect on weaning. & 0.409 & & & & \\
\hline Breast dependence results in poor eating behaviors in the child. & 0.474 & & & & \\
\hline The child should be weaned at any age if he/she is underweight. & 0.713 & & & & \\
\hline The child should be weaned if he/she is not tending to supportive nutrition. & 0.753 & & & & \\
\hline The child should be weaned if the mother has any kind of illness or disease. & 0.566 & & & & \\
\hline The child should be weaned if the mother consumes any kind of drug. & 0.554 & & & & \\
\hline The child should be weaned if the mother becomes pregnant. & 0.485 & & & & \\
\hline $\begin{array}{l}\text { Using disgusting methods like pouring the child's unfavorable drugs or syrups on breast can help with } \\
\text { weaning. }\end{array}$ & & 0.581 & & & \\
\hline $\begin{array}{l}\text { Using traditional and common methods like coloring the breast or pouring pepper on it can help with } \\
\text { weaning. }\end{array}$ & & 0.504 & & & \\
\hline $\begin{array}{l}\text { Using scary methods like drawing on the breast, making it red, or sticking objects on it can help with } \\
\text { weaning. }\end{array}$ & & 0.504 & & & \\
\hline Creating distance between the mother and her child is among the effective methods for weaning. & & 0.611 & & & \\
\hline Talking to and convincing the child is one of the weaning methods. & & 0.413 & & & \\
\hline Expressing the milk and feeding the child with stored milk is a good method for weaning. & & 0.465 & & & \\
\hline Stirring the child's emotions by pretending the breast is painful or wounded is effective in weaning. & & 0.673 & & & \\
\hline Inducing milk that is poisoned or spoiled is effective in weaning. & & 0.769 & & & \\
\hline $\begin{array}{l}\text { Inducing the milk, which makes the child's teeth break or his/her mouth to get smelly, is effective in } \\
\text { weaning. }\end{array}$ & & 0.770 & & & \\
\hline Entertaining and occupying the child is one of the weaning methods. & & 0.675 & & & \\
\hline Using a novel method for weaning each child. & & 0.422 & & & \\
\hline Using a gradual method for weaning is effective. & & 0.634 & & & \\
\hline The mother should seek a pediatrician's comments for weaning. & & & 0.440 & & \\
\hline Searching the Internet is an appropriate way to learn about weaning methods. & & & 0.555 & & \\
\hline Studying related books can help mothers find the right weaning method. & & & 0.676 & & \\
\hline Using family members and friends' experiences is an effective method for weaning. & & & 0.589 & & \\
\hline Weaning is a personal issue and no consultation is necessary. & & & 0.406 & & \\
\hline Family members' help and cooperation are needed for weaning. & & & 0.664 & & \\
\hline Receiving education from health center workers is essential for weaning. & & & 0.417 & & \\
\hline The mother may feel guilty after weaning. & & & & 0.406 & \\
\hline The mother may feel depressed after weaning. & & & & 0.509 & \\
\hline Weaning results in a deep emotional distance between the mother and her child. & & & & 0.590 & \\
\hline Weaning results in a maternal feeling of pride. & & & & 0.405 & \\
\hline Weaning results in peace of mind and physical calmness for the mother. & & & & 0.520 & \\
\hline Weaning results in a severe emotional disorder for the mother. & & & & 0.449 & \\
\hline Weaning is a mental shock for the child. & & & & & 0.723 \\
\hline $\begin{array}{l}\text { Weaning may result in physical problems like anorexia, diarrhea, sleeping disorders, or loss of weight in } \\
\text { the child. }\end{array}$ & & & & & 0.795 \\
\hline Weaning may result in unwanted habits like toe sucking, nail biting, and aggression for the child. & & & & & 0.804 \\
\hline $\begin{array}{l}\text { Weaning may result in psychological reactions like fear, anxiety, immorality, pertinacity, and irritability in } \\
\text { the child. }\end{array}$ & & & & & 0.669 \\
\hline Weaning results in a better weight gain and appetite for the child. & & & & & 0.414 \\
\hline
\end{tabular}


To evaluate internal consistency, Cronbach's alpha was calculated for each item, which gave a summated value of 0.88. In addition, the test-retest method was used for probing stability. The results showed that the scores of the first and second tests were statistically significant $(P$ $<0.001$ and ICC $=0.89$ ), approving the repeatability of the subscales and questionnaire, along with showing high stability (Table 3).

The questionnaire included five subscales like contexts (1-19), solutions (20-31), searching for help (32-38), along with maternal (39-44) and child-related (45-49) outcomes. The scoring scale in this questionnaire was based on the five-point Likert-type scale denoting "strongly agree" with a score of five and "strongly disagree" with a score of one. Questions 2, 3, 8, 10, 16-23, 25-28, 36, 41 , and 44 were scored negatively. The total score varied from 19 to 245, and scores of 19-94, 95-169, and 170-245 represented weak, medium, and positive beliefs toward the weaning phenomenon, respectively.

\section{Discussion and Conclusions}

The present study involved designing and developing a questionnaire with 49 items and five subscales for probing the role of cultural beliefs on weaning. The tool was checked thoroughly for reliability and validity. This questionnaire showed acceptable formal validity and the ratio of content validity to the CVI was also appropriate. Further, exploratory factor analysis was applied to all 49 items to determine construct validity. Furthermore, the KMO scale and Bartlett test approved the factor analysis model. The eigenvalues proved the multifactorial specification of the questionnaire. Each item demonstrated a fair relationship with one of the subscales, and the structural validity degree of mean correlation seemed to be reasonable as indicated by the load factor of each item. A statistically significant relationship was observed between the subscales and the total score of the questionnaire.

Moreover, the reliability details of our study seemed to be encouraging. The reliability of the scale was checked both by the internal consistency and retest methods, therefore, strengthening the reliability of test results. The results revealed that the questionnaire has good internal consistency. However, further analyses using larger scales may show stronger relationships.

Further surveys for using the questionnaire in

Table 3. Results of the Internal Consistency Evaluation of the Questionnaire for Probing the Role of Cultural Beliefs on Weaning

\begin{tabular}{lcccc}
\hline Factor & Subscale & Number & Cronbach $\alpha$ & ICC \\
\hline 1 & Contexts & $19(1-19)$ & 0.79 & 0.89 \\
2 & Solutions & $12(20-31)$ & 0.88 & 0.83 \\
3 & Searching for help & $7(32-38)$ & 0.82 & 0.85 \\
4 & Maternal outcomes & $6(39-44)$ & 0.83 & 0.83 \\
5 & Child-related outcomes & $5(45-49)$ & 0.89 & 0.87 \\
\hline
\end{tabular}

larger cultures and populations are necessary. This questionnaire, which we recommend as a mini-scale, can be used in statistical and practical studies. Moreover, it can be utilized in health systems as a scale for probing and examining mothers' beliefs about weaning. Finally, it can also help in assessing each mother's educational needs regarding choosing the appropriate intervention at the first, second, and third levels of prevention according to the child's needs.

\section{Conflict of Interests}

The authors declare that there are no conflict of interests pertaining to this study.

\section{Ethical Issues}

The Ethics Committee of Babol University of Medical Sciences approved the study. All participants were informed about the purpose of the research and informed written consent was obtained from the participants. Voluntary participation, confidentiality, and anonymity were emphasized as well. To ensure privacy, interviews were carried out in a peaceful and private environment.

\section{Financial Support}

This work was financially supported by Babol University of Medical Sciences.

\section{Acknowledgments}

This project was registered at Ramsar Nursing Care Research Center, Babol University of Medical Sciences with the registration number of MUBABOL.REC.1394.177. We appreciate all the mothers and faculty members who helped us with this research.

\section{References}

1. Sloan S, Gildea A, Stewart M, Sneddon H, Iwaniec D. Early weaning is related to weight and rate of weight gain in infancy. Child Care Health Dev. 2008;34(1):59-64. doi:10.1111/j.1365-2214.2007.00771.x

2. Negayama K, Norimatsu H, Barratt M, Bouville JF. JapanFrance-US comparison of infant weaning from mother's viewpoint. J Reprod Infant Psychol. 2012;30(1):77-91. doi :10.1080/02646838.2011.649473

3. Motee A, Ramasawmy D, Pugo-Gunsam P, Jeewon R. An assessment of the breastfeeding practices and infant feeding pattern among mothers in Mauritius. J Nutr Metab. 2013;2013:243852. doi:10.1155/2013/243852

4. Brand E, Kothari C, Stark MA. Factors related to breastfeeding discontinuation between hospital discharge and 2 weeks postpartum. J Perinat Educ. 2011;20(1):36-44. doi:10.1891/1058-1243.20.1.36

5. Robert E, Coppieters Y, Swennen B, Dramaix M. The reasons for early weaning, perceived insufficient breast milk, and maternal dissatisfaction: Comparative studies in two Belgian regions. Int Sch Res Notices. 2014;2014:678564. doi:10.1155/2014/678564

6. Rosen IM, Krueger MV, Carney LM, Graham JA. Prenatal 
breastfeeding education and breastfeeding outcomes. MCN Am J Matern Child Nurs. 2008;33(5):315-319. doi:10.1097/01.NMC.0000334900.22215.ec

7. Fouts HN. Social and emotional contexts of weaning among bofi farmers and foragers. Ethnology. 2004;43(1):65-81. doi: $10.2307 / 3773856$

8. Gielen UP, Roopnarine JL. Childhood and Adolescence: Cross-Cultural Perspectives and Applications: CrossCultural Perspectives and Applications. ABC-CLIO; 2016.

9. Mané NB, Simondon KB, Diallo A, Marra AM, Simondon F. Early breastfeeding cessation in rural Senegal: causes, modes, and consequences. Am J Public Health. 2006;96(1):139-144. doi:10.2105/ajph.2004.048553

10. Deshpande JD, Giri PA, Phalke DB, Phalke VD, Piyush $\mathrm{K}$, Syed MMA. Socio-cultural practices in relation to breastfeeding, weaning and child rearing among Indian mothers and assessment of nutritional status of children under five in rural India. Australas Med J. 2010; 3(9):618624. doi:10.4066/AMJ.2010.417

11. Yaghobi A, Mohagheghi H, Chegini AA, Mohammadzadeh S. Relationship between attachment styles, nutrition type, (Breastfeeding, dry milk) weaning style (suddenly or gradual) with communication styles interpersonal trust in adulthood. Journal of Ilam University of Medical Sciences. 2014;22(1):16-24. [Persian].

12. Stuebe A. The risks of not breastfeeding for mothers and infants. Rev Obstet Gynecol. 2009;2(4):222-231.

13. Zare P, Mirahmadizadeh A, Sayadi M, Moradi F, Mohammadi S. Pattern of complete weaning and its related factors in Fars Province. Journal of Jahrom University of Medical Sciences. 2013;11(2):27-33.

14. Kruger R, Gericke GJ. A qualitative exploration of rural feeding and weaning practices, knowledge and attitudes on nutrition. Public Health Nutr. 2003;6(2):217-223. doi:10.1079/phn2002419

15. Fouts HN, Hewlett BS, Lamb ME. Weaning and the nature of early childhood interactions among bofi foragers in central Africa. Hum Nat. 2001;12(1):27-46. doi:10.1007/ s12110-001-1012-z

16. Rattray J, Jones MC. Essential elements of questionnaire design and development. J Clin Nurs. 2007;16(2):234-243. doi:10.1111/j.1365-2702.2006.01573.x

17. Teddlie C, Tashakkori A. SAGE Handbook of Mixed Methods in Social \& Behavioral Research. SAGE Publications, Inc; 2011:285-300.

18. Morse JM. Principles of mixed methods and multimethod research design. In: Teddlie C, Tashakkori A, eds. Handbook of Mixed Methods in Social and Behavioral Research. Sage; 2003;1:189-208.

19. Graneheim UH, Lundman B. Qualitative content analysis in nursing research: concepts, procedures and measures to achieve trustworthiness. Nurse Educ Today. 2004;24(2):105112. doi:10.1016/j.nedt.2003.10.001

20. Guba EG, Lincoln YS. Competing paradigms in qualitative research. In: Denzin NK, Lincoln YS, eds. Handbook of qualitative research. London: Sage:105-107.

21. Shali M, Ghaffari F, Joolaee S, Ebadi A. Development and psychometric evaluation of the patient safety violation scale in medical oncology units in Iran. Asian Pac J Cancer Prev. 2016;17(9):4341-4347.

22. Lawshe $\mathrm{CH}$. A quantitative approach to content validity. Pers Psychol. 1975;28(4):563-575. doi:10.1111/j.1744-6570.1975. tb01393.x

23. Waltz CF, Bausell BR. Nursing research: design statistics and computer analysis. Philadelphia: FA Davis Co; 1981.

24. Navabi N, Ghaffari F, Shamsalinia A, Faghani S. Development and validation of evaluation tools of nursing students' clinical pharmacology unit. Drug Healthc Patient Saf. 2016;8:101-109. doi:10.2147/dhps.s110774

25. Knapp TR, Brown JK. Ten measurement commandments that often should be broken. Res Nurs Health. 1995;18(5):465-469. doi:10.1002/nur.4770180511

26. Ghaffari F, Shali M, Shoghi M, Joolaee S. Psychometric properties of the Persian version of the self- assessed support needs questionnaire for breast cancer cases. Asian Pac J Cancer Prev. 2014;15(3):1435-1440. doi:10.7314/ apjcp.2014.15.3.1435

27. Liang Y, Lau PWC, Huang WYJ, Maddison R, Baranowski T. Validity and reliability of questionnaires measuring physical activity self-efficacy, enjoyment, social support among Hong Kong Chinese children. Prev Med Rep. 2014;1:48-52. doi:10.1016/j.pmedr.2014.09.005

(C) 2020 The Author (s); This is an open-access article distributed under the terms of the Creative Commons Attribution License (http://creativecommons.org/licenses/by/4.0), which permits unrestricted use, distribution, and reproduction in any medium, provided the original work is properly cited. 